Editorial

\title{
Recent Developments in Cointegration
}

\author{
Katarina Juselius \\ Department of Economics, University of Copenhagen, DK-1353 Copenhagen, Denmark; \\ katarina.juselius@econ.ku.dk
}

Received: 25 December 2017; Accepted: 28 December 2017; Published: 31 December 2017

As an empirical econometrician, I have always strongly believed in the power of analyzing statistical models as a scientifically viable way of learning from observed data. In the aftermath of the great recession, this seems more important than ever. Most economic and econometric models in macroeconomics and finance did not seem well geared to address features in the data of key importance for the crisis. In particular, the long persistent movements away from long-run equilibrium values typical of the pre-crisis period seem crucial in this respect. Because of this, I hoped that the papers submitted to this special issue would use cointegration to address these important issues, for example by applying cointegration in models with self-reinforcing feed-back mechanisms, or by deriving new tests motivated by such empirical applications, or by dealing with near integration in the I(1) and I(2) model. No doubt, the outcome surpassed my most optimistic expectations. This special issue contains excellent contributions structured around several interconnected themes most of them addressing the above mentioned issues in one way or the other. While some of the papers are predominantly theoretical others are mainly empirical, all of them represent a good mixture of theory and applications. The theoretical papers solve problems motivated by empirical work and the empirical papers address problems using valid statistical procedures. In this sense, the collection of articles represents econometric modelling at its best. As the guest editor, I feel both proud and grateful to be able to write the editorial for so many high quality research papers.

The high quality of the articles is to a significant degree a result of detailed, insightful and very useful referee reports. I would like to take the opportunity to express a deeply felt gratitude to all the reviewers having invested their precious time to check and improve the quality of the articles. Your efforts made all the difference.

How to link a theoretical model with empirical evidence in a scientifically valid way is a tremendously difficult task that has been debated as long as economics and econometrics have existed. The dilemma facing an empirical economist/econometrician is that there are many economic models but only one economic reality: which of the models should be chosen? Rather than choosing one economic model and forcing it onto the data, the CVAR model structures the data based on the likelihood principle to obtain broad confidence intervals within which potentially relevant economic models should fall. This is consistent with the basic ideas of Trygve Haavelmo who, with his Nobel Prize winning monograph "The Probability Approach to Economics", can be seen as the forefather of the modern likelihood based approach to empirical economics. Juselius (2015) argues that the Cointegrated VAR model, by allowing for unit roots and cointegration, provides a solution to some of the statistical problems Trygve Haavelmo struggled with. Hoover and Juselius (2015) argue that a theory consistent CVAR scenario can be interpreted in terms of Haavelmo's notion of an "experimental design for data based on passive observations". A CVAR scenario translates all basic hypotheses of an economic model into a set of testable regularities describing long-run relations and common stochastic trends. If the basic assumptions of the theoretical model are empirically valid, then one should see these regularities in the data. As such scenarios can be formulated for competing models and then checked against data, it can be seen as a scientifically valid way of linking economic models with the statistical data. A theoretical model that passes the first check of such basic properties is potentially an 
empirically relevant model. Examples of CVAR scenarios for various standard economic models can be found in Juselius $(2006,2017)$ and Juselius and Franchi (2007).

To learn about the mechanisms that tend to generate crises, we need furthermore to develop methodological principles that can link economic models consistent with self-reinforcing adjustment behavior to the econometric model (here the CVAR model). My own paper "Using a Theory-Consistent CVAR scenario to test a real exchange rate model based on imperfect knowledge" is an attempt to do so. As such, it also works as a motivating introduction to the main themes of the special issue. It addresses the dilemma of unobserved expectations and the crucial role they play in economic models versus a CVAR model based on observable variables. A solution to this dilemma is obtained by introducing a rather weak assumption on the time-series property of the forecast shock, i.e., the deviation between the actual observation at time $t$ and the value expected at time $t+1$ made at time $t$. Given this assumption, the paper derives a theory-consistent CVAR scenario in which basic assumptions of an imperfect knowledge theory model are translated into testable hypotheses of the CVAR's common stochastic trends and cointegration relations. The derived scenario shows that under the assumption of imperfect knowledge expectations, the data are likely to be near I(2). This is because such expectations tend to drive prices away from long-run equilibrium states for extended periods of time and hence generate long persistent swings in the data. An application to the real exchange rate between Germany and the USA shows a remarkable support for the derived scenario.

The I(2) model has a rich but also more complex structure than the I(1) model. In particular, the computational complexities behind the estimation of the various structures describing the long run, medium run and the short run are quite daunting. The paper by Jurgen Doornik "Maximum Likelihood Estimation of the I(2) Model under Linear Restrictions" discusses how to calculate ML estimates of the CVAR model for I(2) data both in its autoregressive and moving average representation. As ML estimation of I(2) models always requires iteration, the paper discusses different algorithms and offers a new, so called "triangular form", representation of the I(2) model. While it offers an efficient way of calculating the estimates, the triangular form represents a certain mathematical beauty by itself. The algorithm is implemented in the new software package CATS 3 in OxMetrics (Doornik and Juselius 2017) which allows for a full-fledged I(2) procedure that calculates ML tests and estimates of the I(2) model also under linear restrictions.

The recent theory of imperfect knowledge economics offers an economic framework for addressing certain features in the data, such as long persistent swings away from equilibrium values, which often are associated with financial behavior in asset markets. The paper by Leonardo Salazar "Modeling Real Exchange Rate Persistence in Chile" takes as a starting point the long and persistent swings in the real Chilean dollar exchange rate and uses a monetary model based on imperfect knowledge economics as a theoretical explanation for this persistence. Applying the ideas of Juselius (this issue) he finds that the data cannot be rejected as I(2) and that the results give support for the hypothesis of error-increasing behavior in prices and interest rates. He finds that persistent movements in the real exchange rate are compensated by similar movements in the interest rate spread, a result that has also been found in Juselius (this issue) as well as in Juselius (2006) and Juselius and Assenmacher (2017). But in the present case the copper price was also needed to explain the deviations of the real exchange rate from its long-run equilibrium value.

Another field where expectations play an important role for the price setting is in the housing market. This became painfully obvious when excessive movements in house prices kick started the worst recession since the depression in the thirties. The paper by Andreas Hetland and Simon Hetland "Short-Term Expectation Formation Versus Long-Term Equilibrium Conditions: The Danish Housing Market" show that the long-swings behavior observed in the market price of Danish housing can be understood by studying the interplay between short-term expectations and long-run equilibrium conditions. They introduce an asset market model for housing based on imperfect knowledge in which the demand for housing is affected by uncertainty rather than just risk. Under rather mild assumptions, this leads to other forms of forecasting behavior than usually found when assuming so called rational 
expectations. The data were found to be I(2) consistent with imperfect knowledge models. Using the I(2) cointegrated VAR model they find that the long-run equilibrium for the housing price corresponds closely to the predictions from the theoretical model. The results of the paper corroborate previous findings that the housing market is well characterized by short-term momentum forecasting behavior. However, the conclusions have even greater relevance since house prices (through wealth effects) often play an important role in the wider Danish economy, as well as other developed economies.

While it is well known how to estimate the different structures of the I(2) model either unrestrictedly or subject to just-identifying restrictions, it is more difficult to derive tests of over-identifying restrictions on these structures. In particular, ML tests of over-identifying restrictions on the common trends parameters are rare or nonexistent, whereas tests of certain non-identifying hypotheses on the common trends can be found. Common for the latter is that they imply the same restriction on the long-run beta structure and, hence, can easily be translated into hypotheses on the cointegration vectors. When the restrictions are over-identifying, it is much more challenging to derive such test procedures. The paper by Peter Boswijk and Paolo Paruolo "Likelihood Ratio Tests of Restrictions on Common Trends Loading Matrices in I(2) VAR Systems" derives a new test for over-identifying restrictions on the common trends loading matrices in an I(2) model. It shows how a fairly complex over-identifying hypothesis on the common trends loadings matrix can be translated into hypotheses on the cointegration parameters, presents an algorithm for (constrained) maximum likelihood estimation, and provides a sketch of its asymptotic properties. As an illustration, the paper tests an imperfect knowledge hypothesis on the loadings of the common trends discussed in Juselius and Assenmacher (2017) motivated by an analysis of the PPP and UIP between Switzerland and the US. The hypothesis did not obtain empirical support implying that the original hypothesis has to be modified to some extent.

Few economic models were able to foresee the great recession which started a (sometimes heated) debate on the usefulness of standard economic models. Many economists argued that the great recession couldn't possibly have been predicted: it was a black swan. Many empirical econometricians were less convinced, the long and persistent imbalances had been all but invisible in the period preceding the crisis. Mikael Juselius and Moshe Kim demonstrate in their paper "Sustainable Financial Obligations and Crisis Cycles" that an econometric model based on cointegration and smooth transition would have been able to foresee three of the most recent economic crises in USA: the savings and loans crisis in 1992, the IT-bubble crisis in 1995, and the great recession in 2008. In all three cases, the paper shows that debt service burdens in the household and the business sector exceeded their respective estimated sustainable debt level approximately 1-2 years before the recession started. This result is obtained by calculating the sustainable level of debt using the financial obligations ratio as a measure of the incipient aggregate liquidity constraint instead of the often used debt-income ratio. An interesting result is that the intensity of the interaction between credit losses and the business cycle was found to depend on whether the credit losses originate in the household or the business sector. For example, the savings and loans crisis originated primarily from losses in the private household sector, the IT crises from the business sector, and the great recession from both the private and the business sector. As excessive debt is often the main trigger of a financial crisis, exemplified by the recent crises, failure to foresee and prevent it is likely to cause a breakdown of economic stability. In this sense, the results have important implication for how to design macroprudential policy and countercyclical capital-buffers.

Many economists argue correctly that true unit roots are implausible in economic data, as over the very long run this would lead to data properties that are generally not observed: economic data do not tend to move away from equilibrium values forever. With this said, data often contain characteristic roots, which are so close to the unit circle that a standard unit root test would not be able to reject the null of unity. Juselius (in this issue) argues therefore that a unit root should not be considered a structural economic parameter (as is frequently done in the literature), rather one should think of it as a statistical approximation that allows us to structure the data according to their persistency properties. The advantage is that inference can be made about the long-run, the medium-run and the short-run 
in the same model. Still, the question of how this affects the probability analysis of the CVAR model has to be addressed. The paper by Massimo Franchi and Søren Johansen "Improved Inference on Cointegrating Vectors in the Presence of a near Unit Root Using Adjusted Quantiles" takes its starting point from the paper by Elliott (1998). The latter shows that correct inference on a cointegrating relation depends in a complex way on whether the model contains a near unit root trend or not and that the test for a given cointegration vector may have rejection probabilities under the null that deviate from the nominal size as much as $90 \%$. The present paper extends previous results by Elliott (1998) by using a CVAR model with multiple near unit roots. It derives the asymptotic properties of the Gaussian maximum likelihood estimator and a test of a cointegrating relation in a model with a single near unit root using the two critical value adjustments suggested by McCloskey (2017). A simulation study shows that the latter eliminate the above mentioned serious size distortions and demonstrates that the test has reasonable power for relevant alternatives. By analyzing a number of different bivariate Data Generating Processes, the paper shows that the results are likely to hold more generally.

The focus of empirical work is often on estimating and identifying long-run cointegration relations, rather than common stochastic trends. The latter is intrinsically more difficult as common trends are usually assumed to be functions of unobserved structural shocks, whereas the estimated residuals are not structural as they tend to change every time a new variable is added to the model. In spite of the difficulty it is of considerable interest to identify correctly the structural trends as they describe the exogenous forces pushing the economy. Failure to do so opens up for a plethora of interpretations often based on the same data. Since it is natural to estimate unobserved common trends based on unobserved components models, this is the starting point for the paper by Søren Johansen and Morten Nyboe Tabor "Cointegration between Trends and Their Estimators in State Space Models and Cointegrated Vector Autoregressive Models". The state space model with an unobserved multivariate random walk and a linear observation equation is studied with the purpose of finding out under which conditions the extracted random walk trend cointegrates with its estimator. The criterion is that the difference of the two should be asymptotically stationary. The paper shows that this holds for the extracted trend given by the linear observation equation, but no longer when identifying restrictions are imposed on the trend coefficients in the observation equation. Only when the estimators of the coefficients in the observation equation are consistent at a faster rate than the square root of the sample size will there be cointegration between the identified trend and its estimator. The same results hold when data generated from the state space model are analyzed with a cointegrated vector autoregressive model. The findings are illustrated by a small simulation study.

While panel data models with cointegration are widely used, the role of the deterministic terms in the model is still open to debate. This is addressed in the paper by Uwe Hassler and Mehdi Hosseinkouchack "Panel Cointegration Testing in the Presence of Linear Time Trends", which considers a class of panel tests of the null hypothesis of no cointegration when the data contain linear time trends. All tests under investigation rely on single-equations estimated by least squares, and the tests can be either residual-based or not. The focus is on test statistics computed from regressions with intercept only and with at least one of the regressors being dominated by a linear time trend. In such a setting, often encountered in practice, the limiting distributions and critical values provided for the case "with intercept only" are not correct. The paper demonstrates that this leads to size distortions growing with the panel size $N$, reports the appropriate distributions, and shows how correct critical values are obtained.

Today, most econometric packages contain a cointegration routine that calculates estimates and test results using various kinds of algorithms. The more complex the model to be estimated, the more complex is the algorithm to be used. Some algorithms may stop at local maxima, others are more powerful in finding the global maximum. For an applied econometrician, it is a serious problem that the same model applied to the same data may give different results depending on the algorithm used. This is the motivation behind the paper by Jurgen Doornik, Rocco Mosconi, and Paolo Paruolo "Formula I(1) and I(2): Race Tracks for Likelihood Maximization Algorithms of I(1) and 
I(2) Cointegrated VAR Models". It provides a number of test cases, called circuits, for the evaluation of Gaussian likelihood maximization algorithms of the cointegrated vector autoregressive model. Both I(1) and I(2) models are considered. The performance of algorithms is compared first in terms of effectiveness, defined as the ability to find the overall maximum. The next step is to compare their efficiency and reliability across experiments. The aim of the paper is to commence a collective learning project by the profession on the actual properties of algorithms for CVAR model estimation, in order to improve their quality and, as a consequence, the reliability of empirical research.

Conflicts of Interest: The authors declare no conflict of interest.

\section{References}

Doornik, Jurgen, and Katarina Juselius. 2017. Cointegration Analysis of Time Series Using CATS 3 for OxMetrics. Richmond: Timberlake Consultants Ltd.

Elliott, Graham. 1998. On the robustness of cointegration methods when regressors almost have unit roots. Econometrica 66: 149-58. [CrossRef]

Hoover, Kevin, and Katarina Juselius. 2015. Trygve Haavelmo'S Experimental Methodology and Scenario Analysis in a Cointegrated Vector Autoregression. Econometric Theory 31: 249-74. [CrossRef]

Juselius, Katarina. 2006. The Cointegrated VAR Model. Oxford: Oxford University Press.

Juselius, Katarina. 2015. Haavelmo's Probability Approach and the Cointegrated VAR model. Econometric Theory 31: 213-32. [CrossRef]

Juselius, Katarina. 2017. A Theory-Consistent CVAR Scenario: Testing a Rational Expectations Based Monetary Model. Working paper, Department of Economics, University of Copenhagen, Copenhagen.

Juselius, Katarina, and Katrin Assenmacher. 2017. Real exchange rate persistence and the excess return puzzle: The case of Switzerland versus the US. Journal of Applied Econometrics 32: 1145-55. [CrossRef]

Juselius, Katarina, and Massimo Franchi. 2007. Taking a DSGE Model to the Data Meaningfully. Economics-The Open-Access, Open-Assessment E-Journal 1: 1-38. Available online: http:/ /www.economics-ejournal.org/ economics/journalarticles/2007-4 (accessed on 12 June 2017). [CrossRef]

McCloskey, Adam. 2017. Bonferroni-based size-correction for nonstandard testing problems. Journal of Econometrics 200: 17-35. Available online: http://www.sciencedirect.com/science/article/pii/ S0304407617300556 (accessed on 12 June 2017). [CrossRef] 